\title{
The Characteristics and Survival of Patients with Mesorectum Metastatic Lymph Nodes from Cervical Cancer
}

This article was published in the following Dove Press journal: Cancer Management and Research

\section{Weiping Wang (D) \\ Xiaoliang Liu \\ Fuquan Zhang (D* \\ $\mathrm{Ke} \mathrm{Hu}$ (D) $^{*}$}

Department of Radiation Oncology, Peking Union Medical College Hospital, Chinese Academy of Medical Sciences \& Peking Union Medical College, Beijing, People's Republic of China

*These authors contributed equally to this work
Correspondence: Fuquan Zhang; Ke Hu Department of Radiation Oncology, Peking Union Medical College Hospital, Chinese Academy of Medical Sciences \& Peking Union Medical College, No. I Shuaifuyuan Wangfujing, Dongcheng District, Beijing 100730, People's Republic of China

Tel +86-10-69|55485; +86-10-69|55487 Fax +86-10-69155482

Email zhangfq@pumch.cn; huk@pumch.cn
Purpose: To analyze the characteristics and survival of patients with mesorectum metastatic lymph nodes (MLNs) from cervical cancer.

Patients and methods: We retrospectively reviewed 1194 consecutive patients with FIGO stage IA-IVA cervical cancer who were treated with definitive radiotherapy between January 2011 and December 2015 in our institute. Patients were treated with intensitymodulated radiation therapy (IMRT) and brachytherapy, combined with concurrent chemotherapy. Mesorectum, pelvic and para-aortic MLNs were boosted 59-61Gy with IMRT. We calculated the clinical characteristics and survival of the patients with and without mesorectum MLNs and made comparisons between them.

Results: The incidence rate of mesorectum MLNs was $0.8 \%$ (10/1194). The incidence rates for patients with FIGO stages I, II, III, and IVA disease were $0 \%, 0.4 \%, 2.0 \%$, and $33.3 \%$, respectively. Mesorectum MLNs were associated with more advanced stage $(\mathrm{p}<0.001)$; larger tumor size $(\mathrm{p}=0.002)$, para-aortic MLNs $(\mathrm{p}<0.001)$, common iliac MLNs ( $\mathrm{p}$ $<0.001$ ), and bilateral pelvic MLNs ( $\mathrm{p}<0.001$ ). All patients with mesorectum MLNs experienced treatment failure and died during follow-up. The median overall survival was 10.43 months. The 2-year overall survival (OS), disease-free survival (DFS), and local control (LC) rates were $10 \%, 0 \%$, and $30 \%$, respectively. Multivariate analysis showed that that mesorectum MLNs (hazard ratio, HR 4.0, 95\% CI 1.8-9.1, $\mathrm{p}=0.001$ ), para-aortic MLNs (HR 1.8, 95\% CI 1.1-2.8, p=0.017) and pelvic MLNs (HR 2.5, 95\% CI 1.7-3.7, $\mathrm{p}<0.001)$ were independent prognostic factors of OS.

Conclusion: Mesorectum MLNs from cervical cancer are rare. And, the survival of patients with mesorectum MLNs was poor after dose-escalated concurrent chemoradiotherapy.

Keywords: uterine cervical neoplasms, mesorectum lymph nodes, radiotherapy

\section{Introduction}

As a result of effective screening, the incidence rate of cervical cancer has decreased dramatically in developed countries. However, cervical cancer remains a serious issue for women in developing countries such as China. ${ }^{1}$ Lymph node metastases are the most important transport pathways of cervical cancer. It commonly occurs in internal iliac, external iliac, obturator, and common iliac lymph nodes. $^{2-4}$

Mesorectum metastatic lymph nodes (MLNs) are very common in rectum cancer patients, and total mesorectal excision has become the standard approach in the surgery of rectum cancer. For cervical cancer, mesorectum MLNs are rare 
and literature reports are limited. ${ }^{2,5}$ In this study, we retrospectively analyzed the characteristics and survival of patients with mesorectum MLNs in our institute. To our knowledge, this is the first report on the incidence of mesorectum MLNs from cervical cancer and the first report on the survival of these patients.

\section{Materials and Methods Patients}

We searched the electronic database of our institute and retrospectively reviewed records for cervical cancer patients between January 2011 and December 2015. We identified 1194 consecutive patients with International Federation of Gynecology and Obstetrics (FIGO) stages IA-IVA cervical cancer who were treated with definitive radiotherapy or concurrent chemoradiotherapy (CCRT). Of them, 10 patients had mesorectum MLNs before treatment. The criteria for MLNs were as follows: (1) a short diameter longer than $1 \mathrm{~cm}$ on computed tomography (CT) or magnetic resonance (MR) images and (2) confirmed by positron emission tomography/computed tomography (PET/CT). The mesorectum region was the area within the mesorectum, with an upper bound in the level of the lower edge of the sacroiliac joints. Involved lymph nodes in the mesorectum region were defined as mesorectum MLNs. Lymph nodes above the lower edge of the sacroiliac joints were grouped into presacral nodes.

The clinical characteristics of all patients (including patients with or without mesorectum MLNs) were collected, including age, histology, FIGO stage, the number and location of regional MLNs, the diagnostic modalities for mesorectum MLNs, and treatment information.

\section{Treatment}

Patients were treated with CCRT as described previously. ${ }^{6,7}$ All patients received CT simulation. Gross tumor volume (GTV) and clinical target volume (CTV) were contoured on the CT slices. The GTV covered the regional MLNs, and the CTV included the primary tumor, cervix, parametrium, and pelvic lymph node regions. The para-aortic lymph node region was included in the CTV for patients with paraaortic MLNs or high risk of para-aortic region failure. For patients with mesorectum MLNs, part of the mesorectum region was also included in the CTV. A margin of $5 \mathrm{~mm}$ was added to the GTV to generate the planning gross tumor volume (PGTV). The CTV was expanded $6-10 \mathrm{~mm}$ to create the planning clinical target volume (PCTV). A dose of $50.4 \mathrm{~Gy}$ in 28 fractions was prescribed to the planning target volume (PTV), and 59-61 Gy was delivered to the MLNs with a simultaneous-integrated boost (SIB) with intensity-modulated radiation therapy (IMRT). High-doserate intracavitary brachytherapy (ICBT) began after 3 weeks of external beam radiotherapy. A dose of 30-36 Gy in 5-7 fractions was delivered to point $\mathrm{A}$. The first-line regimen of concurrent chemotherapy was cisplatin $30-40 \mathrm{mg} / \mathrm{m}^{2}$ weekly.

\section{Follow-Up and Statistics}

As prescribed previously, ${ }^{6,7}$ patients had gynecological examinations and pelvic MRI/CT 1 month after treatment. Subsequently, patients had follow-up examinations every 3 months for the next 2 years, every 6 months for 3-5 years, and once a year for 5 years after that. For patients with suspicious recurrence tumor, biopsy and/or were recommended.

Overall survival (OS), disease-free survival (DFS), and local control (LC) were estimated using the Kaplan-Meier method. The significance of difference was examined with a log-rank test. Cox's regression model was used for multivariate analysis. We also compared the characteristics of patients with and without mesorectum MLNs with a continuity-correction Chi-square test (histology, paraaortic MLNs, common iliac MLNs, and treatment approach), Fisher's exact test (treatment approach), $t$-tests (age and tumor size), and Mann-Whitney test (FIGO stage). Statistical analysis was performed using SPSS v.22.0. Difference was considered statistically significant at twosided $\mathrm{p}<0.05$.

\section{Results}

The incidence of mesorectum MLNs was 0.8\% (10/1194) for cervical cancer patients. The detailed clinical characteristics and survival of patients with mesorectum MLNs are summarized in Table 1. The MRI T2 and diffusion-weighted imaging (DWI) images of patient 10 in Table 1 are shown in Figure 1, and the PET/CT images of patient 4 in Table 1 are shown in Figure 2. The median age was 50 years (range, $37-63$ years). Nine patients had squamous cell carcinoma, and 1 patient had adenocarcinoma. Three patients had stage IIB, 4 patients had stage IIIB, and 3 patients had stage IVA disease. The incidence rates for patients with FIGO stages I, II, III, and IVA disease were $0 \%(0 / 154), 0.4 \%$ (3/826), 2.0\% (4/205), and 33.3\% (3/9), respectively. In patients with FIGO III-IV disease and pelvic MLNs, the incidence of 


\begin{tabular}{|c|c|c|c|c|c|c|c|c|c|c|c|}
\hline \multicolumn{2}{|c|}{ 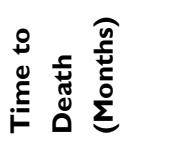 } & $\underset{\sigma}{\stackrel{N}{a}}$ & $\begin{array}{l}\text { ㅇ } \\
0 \\
0\end{array}$ & 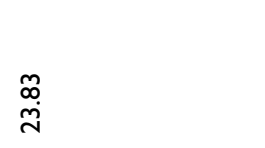 & $\underset{\infty}{\stackrel{m}{\infty}}$ & 오․ & $\begin{array}{l}\text { f. } \\
\stackrel{1}{0}\end{array}$ & ì & 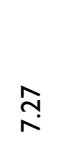 & $\begin{array}{l}\hat{n} \\
\stackrel{n}{0}\end{array}$ & $\begin{array}{l}\text { \&. } \\
\text { ᄋे }\end{array}$ \\
\hline \multicolumn{2}{|c|}{ 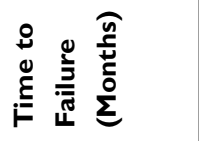 } & $\underset{\substack{n \\
i}}{n}$ & $\underset{f}{\stackrel{F}{*}}$ & $\stackrel{\substack{\infty \\
\sim}}{.}$ & $\begin{array}{l}\hat{\mathrm{I}} \\
.\end{array}$ & ㅇ. & $\stackrel{\circ}{\circ}$ & $\stackrel{8}{\circ}$ & ㅇ. & ¿: & గ̂nુ \\
\hline \multicolumn{2}{|l|}{ 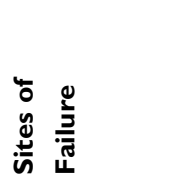 } & 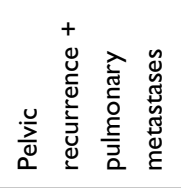 & 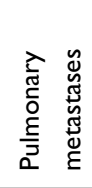 & 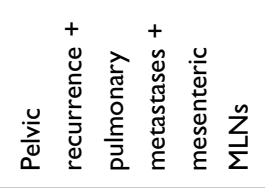 & 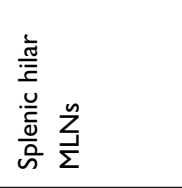 & $\stackrel{\alpha}{0}$ & $\stackrel{\alpha}{\alpha}$ & $\stackrel{\mathscr{a}}{\alpha}$ & $\stackrel{\alpha}{\alpha}$ & $\stackrel{\alpha}{\alpha}$ & 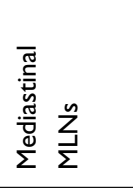 \\
\hline \multicolumn{2}{|l|}{ 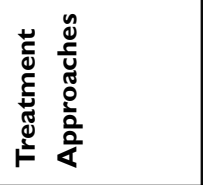 } & 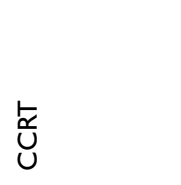 & 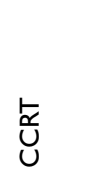 & 矛 & 突 & 预 & b & 窎 & 虞 & 感 & 它 \\
\hline \multicolumn{2}{|l|}{ 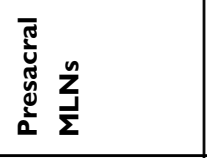 } & $\stackrel{\circ}{z}$ & $\stackrel{\circ}{z}$ & $\stackrel{\circ}{z}$ & $\stackrel{\tilde{\nu}}{\check{\nu}}$ & $\stackrel{\tilde{u}}{\tau}$ & $\stackrel{\mathscr{\Perp}}{\tau}$ & $\stackrel{\mathscr{\nu}}{\check{\nu}}$ & $\stackrel{\tilde{\nu}}{\check{\nu}}$ & $\stackrel{0}{z}$ & \& \\
\hline \multicolumn{2}{|c|}{ 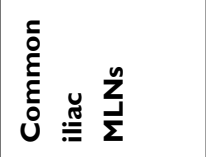 } & $\stackrel{\tilde{\nu}}{\nu}$ & $\stackrel{\mathscr{y}}{\nu}$ & $\stackrel{\tilde{\nu}}{\check{\nu}}$ & ㅇ & $\stackrel{\tilde{\nu}}{\nu}$ & $\stackrel{\mathscr{\nu}}{\check{\nu}}$ & $\stackrel{\tilde{y}}{\check{\nu}}$ & $\stackrel{\tilde{\nu}}{\check{\nu}}$ & $\stackrel{\tilde{\nu}}{\check{\nu}}$ & z \\
\hline \multicolumn{2}{|l|}{ 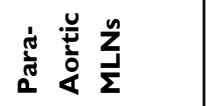 } & $\stackrel{\tilde{\nu}}{\check{\nu}}$ & $\stackrel{\circ}{z}$ & $\stackrel{\tilde{\nu}}{\sim}$ & $\stackrel{y}{\nu}$ & $\stackrel{\tilde{\nu}}{\check{\nu}}$ & 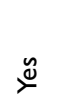 & $\stackrel{\tilde{v}}{\check{\nu}}$ & $\underbrace{\tilde{y}}$ & $\stackrel{\check{\nu}}{\check{\nu}}$ & 으 \\
\hline \multirow{3}{*}{ 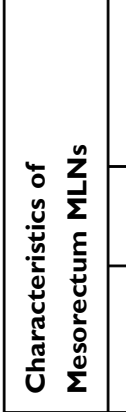 } & 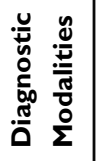 & $\stackrel{5}{\underline{w}}$ & $\overline{\underline{\Sigma}}$ & $\overline{\underline{\Sigma}}$ & 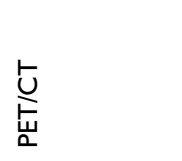 & $\stackrel{\leftarrow}{ }$ & $\stackrel{\bullet}{u}$ & $\begin{array}{l}\sum \\
\frac{ \pm}{\alpha} \\
\frac{\dot{\alpha}}{\Sigma}\end{array}$ & $\begin{array}{l}\stackrel{5}{\longleftarrow} \\
\stackrel{\rightleftarrows}{a}\end{array}$ & $\mathfrak{u}$ & 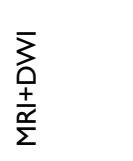 \\
\hline & ज̃ $\widehat{E}$ & 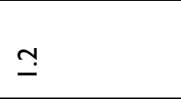 & - & $\stackrel{m}{=}$ & 戸 & = & $\stackrel{+}{ \pm}$ & $\simeq$ & $\stackrel{m}{\underline{m}}$ & $\stackrel{\sim}{\sim}$ & $=$ \\
\hline & 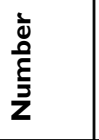 & - & - & - & $\sim$ & - & $N$ & + & N & - & - \\
\hline \multicolumn{2}{|c|}{ 产 } & 0 & นn & $\circ$ & $\circ$ & in & $\infty$ & นn & $m$ & $\wedge$ & + \\
\hline \multicolumn{2}{|l|}{ 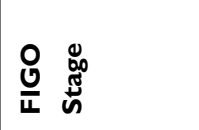 } & $\cong$ & $\stackrel{\varrho}{\equiv}$ & $\stackrel{\varrho}{\equiv}$ & 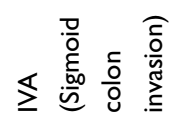 & $\stackrel{\varrho}{\equiv}$ & $\cong$ & $\cong$ & $\stackrel{\varrho}{\equiv}$ & 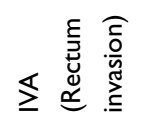 & 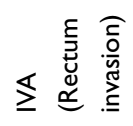 \\
\hline \multicolumn{2}{|l|}{ 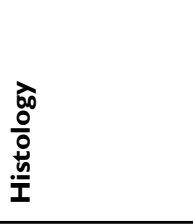 } & ư & ư & $\stackrel{u}{u}$ & ư & $\stackrel{U}{u}$ & ư & 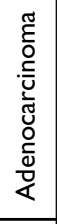 & $\stackrel{u}{u}$ & U. & ư \\
\hline \multicolumn{2}{|l|}{ 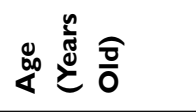 } & 우 & 유 & นn & $\stackrel{\infty}{\sim}$ & $\stackrel{\infty}{+}$ & $\tilde{o}$ & 오 & 요 & $\hat{m}$ & $\mathscr{\sim}$ \\
\hline \multicolumn{2}{|l|}{ z } & - & $N$ & $m$ & $\sigma$ & in & 0 & $n$ & $\infty$ & $\sigma$ & 으 \\
\hline
\end{tabular}




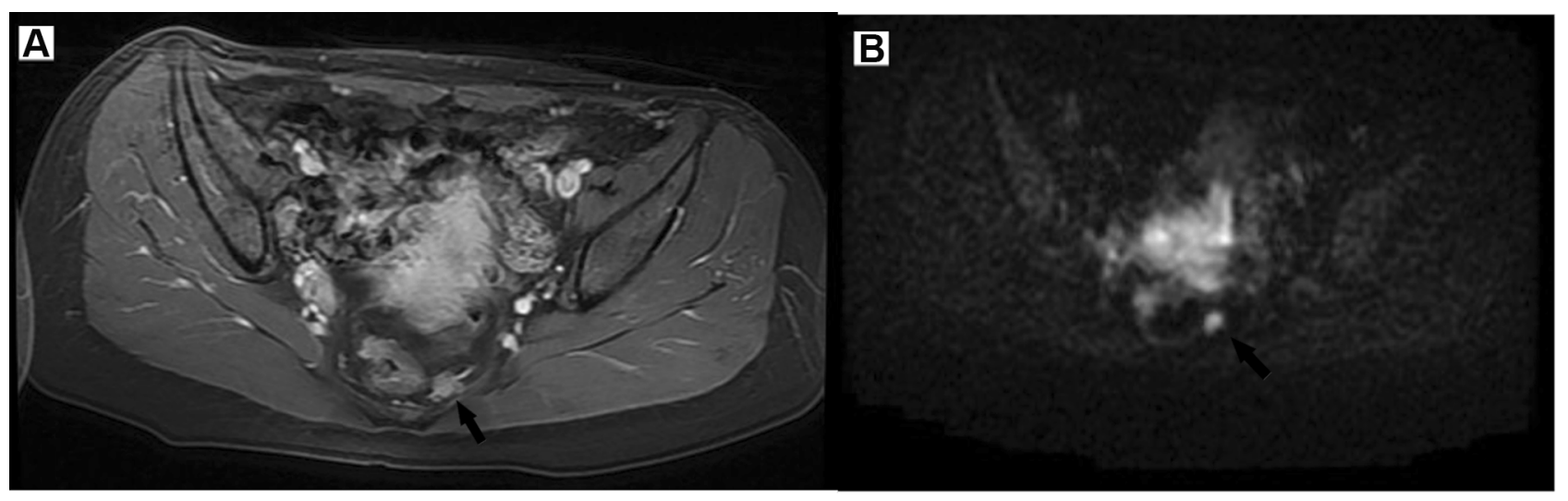

Figure I The T2 weighted-MR (A) and diffusion-weighted imaging (DWI, B) images of a patient with mesorectum metastatic lymph node.

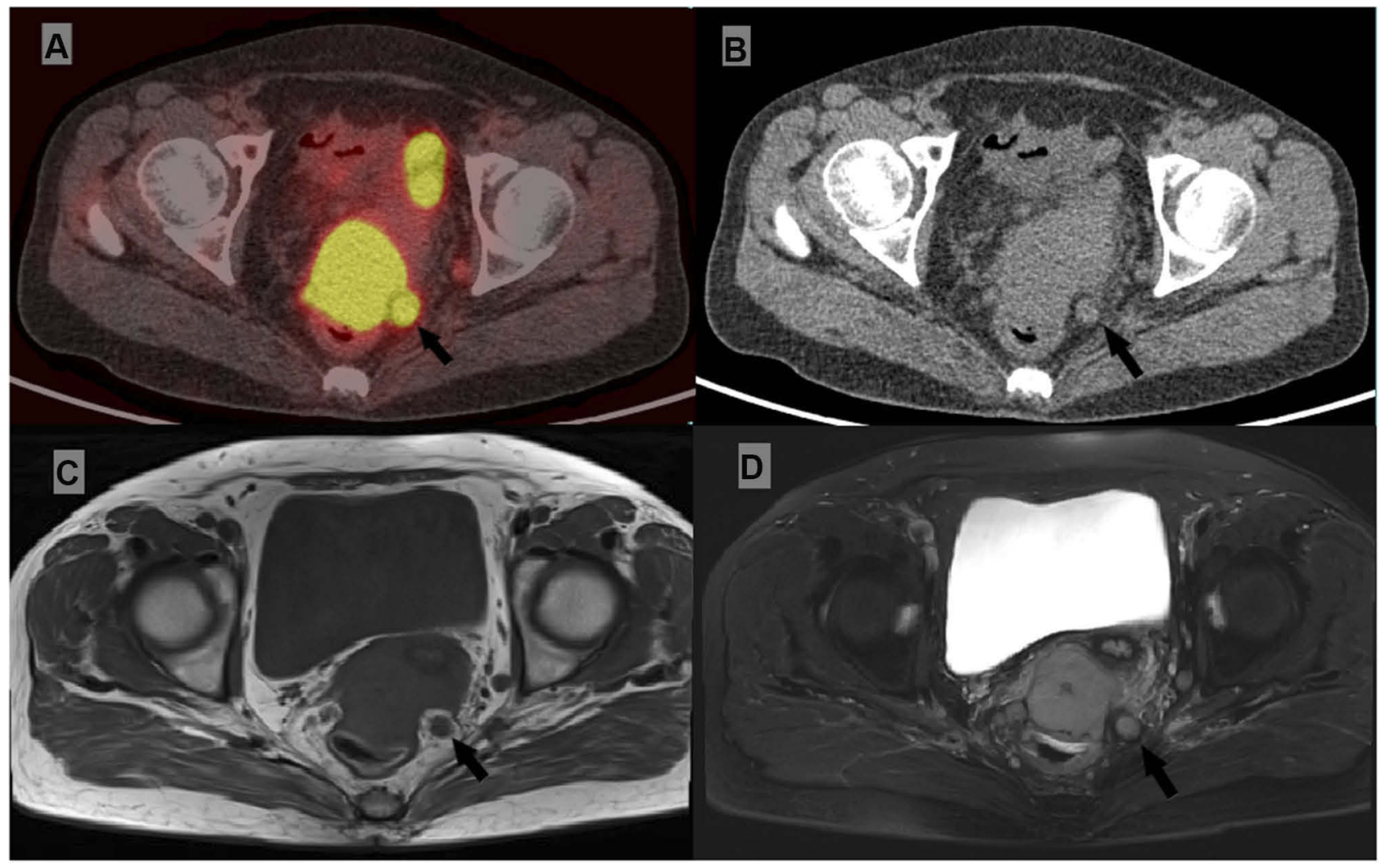

Figure 2 Positron emission tomography/computed tomography (PET/CT, A and B), TI-weighted MR (C) and T2-weighted MR (D) images of a patient with mesorectum metastatic lymph node.

mesorectum MLNs was 3.3\% (7/214) and 2.8\% (10/353), respectively. The median size of the tumor was $6 \mathrm{~cm}$ (range, 3-8 cm). Mesorectum MLNs were diagnosed by PET/CT in 3 patients, MRI in 4 patients, and contrast-enhanced computed tomography (CT) in 3 patients. A total of 16 mesorectum MLNs were found in these 10 patients. The numbers of mesorectum MLNs were 1 in 6 patients, 2 in 3 patients, and 4 in 1 patient. Eight patients had para-aortic MLNs, and 8 patients had common iliac MLNs. All 10 patients suffered with bilateral pelvic MLNs, and 5 of them had presacral MLNs. All 10 patients completed radiotherapy. The fractionated doses of ICBT were 36 Gy in 6 fractions in 5 patients, $30 \mathrm{~Gy}$ in 5 fractions in 4 patients, and $34 \mathrm{~Gy}$ in 6 fractions in 1 patient. Concurrent chemotherapy was administered in 9 patients. Of them, 5 times of cisplatin were performed in 5 patients, 4 times in 2 patients, and 3 times in 2 patients. 
Table 2 The Clinical Characteristics of Patients with and Without Mesorectum MLNs

\begin{tabular}{|c|c|c|c|c|c|}
\hline \multirow[t]{2}{*}{ Characteristics } & \multicolumn{2}{|c|}{ With Mesorectum MLNs $(n=10)$} & \multicolumn{2}{|c|}{ Without Mesorectum MLNs $(n=1184)$} & \multirow[t]{2}{*}{$\mathbf{p}$} \\
\hline & $\mathbf{n}$ & Percentage (\%) & $\mathbf{n}$ & Percentage (\%) & \\
\hline \multicolumn{6}{|c|}{ Median age (years old) } \\
\hline & \multicolumn{2}{|c|}{50 (range, 37-63) } & \multicolumn{2}{|c|}{54 (range, 23-88) } & 0.271 \\
\hline \multicolumn{6}{|l|}{ Histology } \\
\hline SCC & 9 & 90 & 1056 & 89.2 & 1.000 \\
\hline Non-SCC & I & 10 & 128 & 10.8 & \\
\hline \multicolumn{6}{|l|}{ FIGO stage } \\
\hline I & 0 & 0 & 154 & 13.0 & $<0.001$ \\
\hline II & 3 & 30 & 823 & 69.5 & \\
\hline III & 4 & 40 & 201 & 17.0 & \\
\hline IVA & 3 & 30 & 6 & 0.5 & \\
\hline \multicolumn{6}{|c|}{ Median tumor size $(\mathrm{cm})$} \\
\hline & \multicolumn{2}{|c|}{6 (range, 3-8) } & \multicolumn{2}{|c|}{4 (range, $0-12$ ) } & 0.002 \\
\hline \multicolumn{6}{|l|}{ Para-aortic MLNs } \\
\hline Yes & 8 & 80.0 & 73 & 6.2 & $<0.001$ \\
\hline No & 2 & 20.0 & 1111 & 93.8 & \\
\hline \multicolumn{6}{|l|}{ Common iliac MLNs } \\
\hline Yes & 8 & 80.0 & 88 & 7.4 & $<0.001$ \\
\hline No & 2 & 20.0 & 1096 & 92.6 & \\
\hline \multicolumn{6}{|l|}{ Bilateral pelvic MLNs } \\
\hline Yes & 10 & 100.0 & 199 & 16.8 & $<0.001$ \\
\hline No & 0 & 0.0 & 985 & 83.2 & \\
\hline \multicolumn{6}{|l|}{ Treatment approach } \\
\hline RT & 1 & 10.0 & 176 & 14.8 & 1.000 \\
\hline CCRT & 9 & 90.0 & 1008 & 85.2 & \\
\hline
\end{tabular}

Abbreviations: MLNs, metastatic lymph nodes; SCC, squamous cell carcinoma.

As shown in Table 2, mesorectum MLNs were associated with more advanced stages $(\mathrm{p}<0.001)$, larger tumor size $(p=0.002)$, higher incidence rates of para-aortic MLNs ( $<<0.001$ ), and common iliac MLNs $(p<0.001)$ than in patients without mesorectum MLNs. Age, histology, and treatment of these two groups were similar.

The median follow-up periods were 26.2 months (range, 1.2-74.1 months) for the whole cohort (1194 patients) and 10.48 months (range, 5.07-28.43 months) for patients with mesorectum MLNs (10 patients). All patients experienced treatment failure and died during follow-up. The median OS was 10.43 months $(95 \%$ confidence interval (CI): 9.25-11.61 months). The 1-year OS, DFS, and LC rates were $30.0 \%, 10.0 \%$, and $30 \%$, respectively. The 2-year OS, DFS, and LC rates were $10 \%, 0 \%$, and $30 \%$, respectively. The OS, DFS, and LC curves of patients with mesorectum MLNs are shown in Figure 3.
For 10 patients with mesorectum MLNs, 5 patients acquired a complete clinical response (CR), and the other 5 patients had a partial clinical response (PR) after treatment. Three of the 5 patients with acquired $\mathrm{CR}$

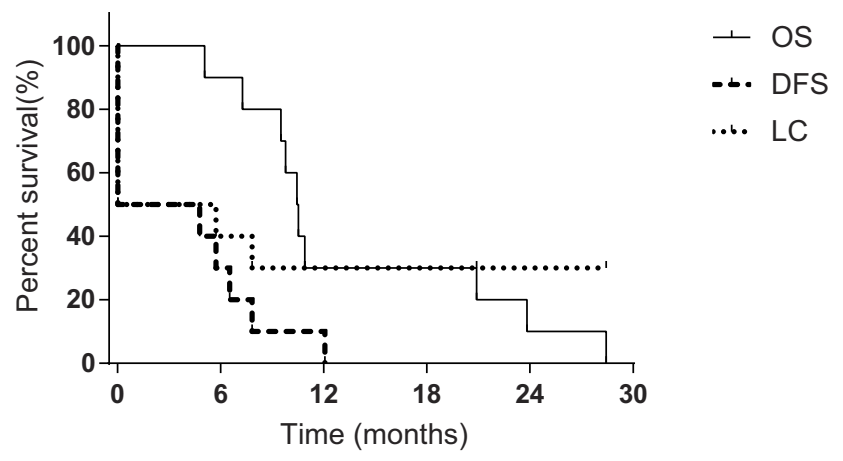

Figure 3 The overall survival (OS), disease-free survival (DFS), and local control (LC) curve of patients with mesorectum metastatic lymph nodes. 
experienced distant metastases, and the other 2 patients had concurrent local recurrence and distant failure. The sites of distant failure included pulmonary metastases (2 patients), mesenteric MLNs (1 patient), mediastinal MLNs (1 patient), and splenic hilar MLNs (1 patient). The pelvic failure and distant failure rates were $70.0 \%$ and $11.6 \%$ $(\mathrm{p}<0.001), 50 \%$ and $15.3 \%(\mathrm{p}<0.001)$ in patients with/ without mesorectum MLNs, respectively.

Univariate analysis showed that age, histology, tumor size, FIGO stage, mesorectum MLNs, para-aortic MLNs, pelvic MLNs, common iliac MLNs, bilateral pelvic MLNs and concurrent chemotherapy were prognostic factors of OS. After multivariate analysis, mesorectum MLNs were an independent prognostic factor of OS (hazard ratio, HR $4.0,95 \%$ CI $1.8-9.1, \mathrm{p}=0.001$ ). The details of univariate and multivariate analysis are shown in Table 3.

For the 73 patients with para-aortic MLNs, the 2-year OS, DFS, and LC rates were $51.3 \%, 40.0 \%$, and $53.3 \%$, respectively, which was also much higher than in patients with mesorectum MLNs $(p=0.004$ for OS rate, 0.001 for DFS rate, and 0.069 for LC rate). For nine patients with FIGO IVA disease, the median OS was 28.43 months (95\% CI 13.0-43.9 months), which was significantly higher than that of patients with mesorectum MLNs $(\mathrm{p}=0.009)$.

In the present study, there were seven patients with rectum invasion. Three of them had mesorectum MLNs and the other four did not have. In patients with and without mesorectum MLNs, the mean DFS was 3.5 months (95\% CI $0-13.0$ months) and 9.5 months (0.7-37.9 months, $\mathrm{p}=0.171)$, respectively. And, the mean LC was 19.0 months (95\% CI 3.8-34.2 months) and 29.1 months (11.3-46.9 months, $\mathrm{p}=0.806)$, respectively. There was a trend that patients with mesorectum MLNs had worse DFS and LC. However, as the number of patients was small, the differences were not significant.

Only 1 patient (patient No. 4 in Table 1) developed grade 3 chronic enteritis, and no other grade 3 or greater chronic toxicity was observed.

\section{Discussion}

Mesorectum MLNs from cervical cancer are rare and studies are limited. In 2016, Barrett et al reported two patients with mesorectum MLNs from advanced cervical cancer. These two patients were also treated with radiotherapy. ${ }^{5}$ As we know, it is the first report on mesorectum MLNs from cervical cancer. Previously, we evaluated the regional lymph nodes of 244 stage IIB cervical cancer patients and found that 1 patient had positive
Table 3 Univariate and Multivariate Analysis for Patients with Cervical Cancer

\begin{tabular}{|c|c|c|c|c|c|}
\hline \multirow[t]{2}{*}{ Characteristics } & \multirow[t]{2}{*}{$\mathbf{N}$} & \multicolumn{2}{|c|}{$\begin{array}{l}\text { Univariate } \\
\text { Analysis }\end{array}$} & \multicolumn{2}{|c|}{ Multivariate Analysis } \\
\hline & & $\begin{array}{l}\text { 3-year } \\
\text { Os }\end{array}$ & $\mathbf{p}$ & $\begin{array}{l}\text { HR }(95 \% \\
\mathrm{Cl})\end{array}$ & $\mathbf{p}$ \\
\hline $\begin{array}{l}\text { Age } \\
\qquad 665 \\
\geq 65\end{array}$ & $\begin{array}{l}1055 \\
139\end{array}$ & $\begin{array}{l}84.6 \% \\
75.1 \%\end{array}$ & 0.014 & $\begin{array}{l}\text { I } \\
1.7(1.1-2.7)\end{array}$ & 0.011 \\
\hline $\begin{array}{l}\text { Histology } \\
\text { SCC } \\
\text { Non-SCC }\end{array}$ & $\begin{array}{l}1065 \\
129\end{array}$ & $\begin{array}{l}84.9 \% \\
71.5 \%\end{array}$ & $<0.001$ & $\begin{array}{l}\text { I } \\
2.3(1.6-3.5)\end{array}$ & $<0.001$ \\
\hline $\begin{array}{l}\text { Tumor size } \\
\quad<4 \mathrm{~cm} \\
\geq 4 \mathrm{~cm}\end{array}$ & $\begin{array}{l}477 \\
717\end{array}$ & $\begin{array}{l}90.3 \% \\
79.0 \%\end{array}$ & $<0.001$ & $\begin{array}{l}\text { I } \\
1.7(1.2-2.5)\end{array}$ & 0.004 \\
\hline $\begin{array}{l}\text { FIGO stage } \\
\text { I } \\
\text { II } \\
\text { III } \\
\text { IVA }\end{array}$ & $\begin{array}{l}154 \\
826 \\
205 \\
9\end{array}$ & $\begin{array}{l}90.8 \% \\
85.3 \% \\
72.7 \% \\
21.4 \%\end{array}$ & $<0.001$ & $\begin{array}{l}1 \\
1.6(0.8-3.1) \\
2.8(1.4-5.7) \\
1.8(0.5-6.9)\end{array}$ & $\begin{array}{l}0.144 \\
0.003 \\
0.362\end{array}$ \\
\hline $\begin{array}{l}\text { Mesorectum } \\
\text { MLNs } \\
\text { Yes } \\
\text { No }\end{array}$ & $\begin{array}{l}1184 \\
10\end{array}$ & $\begin{array}{l}0 \% \\
84.4 \%\end{array}$ & $<0.001$ & $\begin{array}{l}4.0(1.8-9.1) \\
1\end{array}$ & 0.001 \\
\hline $\begin{array}{l}\text { Para-aortic MLNs } \\
\text { Yes } \\
\text { No }\end{array}$ & $\begin{array}{l}81 \\
1113\end{array}$ & $\begin{array}{l}44.0 \% \\
86.3 \%\end{array}$ & $<0.001$ & $\begin{array}{l}1.8(1.1-2.8) \\
1\end{array}$ & 0.017 \\
\hline $\begin{array}{l}\text { Pelvic MLNs } \\
\text { Yes } \\
\text { No }\end{array}$ & $\begin{array}{l}353 \\
841\end{array}$ & $\begin{array}{l}69.3 \% \\
89.2 \%\end{array}$ & $<0.001$ & $\begin{array}{l}2.5(1.7-3.7) \\
1\end{array}$ & $<0.001$ \\
\hline $\begin{array}{l}\text { Common iliac } \\
\text { MLNs } \\
\text { Yes } \\
\text { No }\end{array}$ & $\begin{array}{l}96 \\
1098\end{array}$ & $\begin{array}{l}52.0 \% \\
86.1 \%\end{array}$ & $<0.001$ & $\begin{array}{l}1.5(0.95-2.3) \\
1\end{array}$ & 0.082 \\
\hline $\begin{array}{l}\text { Bilateral pelvic } \\
\text { MLNs } \\
\text { Yes } \\
\text { No }\end{array}$ & $\begin{array}{l}209 \\
985\end{array}$ & $\begin{array}{l}63.5 \% \\
87.6 \%\end{array}$ & $<0.001$ & $\begin{array}{l}1.0(0.7-1.6) \\
1\end{array}$ & 0.938 \\
\hline $\begin{array}{l}\text { Treatment } \\
\text { approach } \\
\text { RT } \\
\text { CCRT }\end{array}$ & $\begin{array}{l}177 \\
1017\end{array}$ & $\begin{array}{l}74.4 \% \\
85.0 \%\end{array}$ & 0.001 & $\begin{array}{l}\text { I } \\
0.6(0.4-0.9)\end{array}$ & 0.010 \\
\hline
\end{tabular}

Abbreviations: MLNs, metastatic lymph nodes; SCC, squamous cell carcinoma.

mesorectum MLNs and 1 patient had suspicious mesorectum MLNs. ${ }^{2}$ In our study, the incidence rate of mesorectum MLNs was $0.8 \%$ (10/1194). To our knowledge, this was the first report on the incidence of mesorectum MLNs in cervical cancer patients. 
The pathway of mesorectum MLNs from cervical cancer is unclear. Probably, it is associated with invasion of the rectum or mesorectum. For patients with FIGO stages I, II, III, and IVA cervical cancer, the incidence rates of mesorectum MLNs were $0 \%, 0.4 \%, 2.0 \%$, and $33.3 \%$, respectively, in our study, and mesorectum MLNs were associated with more advanced FIGO stage $(\mathrm{p}<0.001)$. For patients with FIGO IVA disease, mesorectum MLNs were not rare, especially in patients with rectum mucosa invasion. According to the 2009 FIGO staging system, ${ }^{8}$ when the tumor invades the mesorectum or rectum, and the rectum mucosa is not involved, the tumor is not defined as FIGO stage IVA disease. It was difficult for us to identify the mesorectum invasion accurately on CT, MRI, and PET/CT, and we could not analyze the association between mesorectum invasion and mesorectum MLNs. We also found that patients with mesorectum MLNs were more likely to have para-aortic MLNs ( $\mathrm{p}<0.001$ ), common iliac MLNs $(\mathrm{p}<0.001)$, and bilateral pelvic MLNs $(\mathrm{p}<0.001)$. Refluent lymphatic drainage from the superior mesenteric artery and iliac lymph node regions might be another pathway of mesorectum lymph nodes metastasis. It was reported that the incidence rate of presacral MLNs was just 5\% (6/114) in patients with pelvic MLNs. ${ }^{9}$ In the study by Liu Z, only 1 of 244 patients $(0.4 \%)$ with FIGO stage IIB cervical cancer had positive presacral MLNs. ${ }^{2}$ In the present study, 5 of the 10 patients $(50 \%)$ with mesorectum MLNs had presacral MLNs. It indicated the association between mesorectum MLNs and presacral MLNs in patients with cervical cancer. Considering FIGO stage is closely related with MLNs, the association between mesorectum MLNs and advanced FIGO stage/multiple MLNs should be interpreted with caution.

All patients with mesorectum MLNs experienced treatment failure and died during follow-up. The median OS was just 10.43 months (95\% CI: 9.25-11.61 months). The survival was even much lower than patients with paraaortic MLNs and patients with FIGO stage IVA disease. Para-aortic MLNs and FIGO IVA disease were considered to be the worst prognostic factors for local advanced cervical cancer. This is also the first report on the survival of patients with mesorectum MLNs. The FIGO staging system $^{8}$ did not definitely define that mesorectum MLNs should be classified with regional MLNs or distant metastases. The survival of cervical cancer patients with mesorectum MLNs was poor.

In this study, mesorectum MLNs were diagnosed by imaging. It was reported that the sensitivity of CT, MRI, and PET was $50-59 \%, 56-59 \%$, and $76-82 \%$, respectively, and the specificity was $91-92 \%, 90-91 \%$, and 94-95\%, also, respectively. ${ }^{10,11}$ Considering the comparatively low sensitivity of MLNs in these imaging modalities, we might miss some occult MLNs and underestimate the incidence rate of mesorectum MLNs. However, the specificity of all three imaging modalities was higher than $90 \%,{ }^{10,11}$ and the misdiagnosis rate of our study was low. Transrectal fine needle aspiration cytology is an approach to confirm suspicious mesorectum MLNs. And studies based on histology are more accurate in the diagnose of mesorectum MLNs. This study demonstrated that mesorectum was an independent factor of OS for patients with cervical cancer. Even so, mesorectum MLNs were not the only reason for the poor survival of these 10 patients in the present study. The other factors, such as advanced stage, para-aortic MLNs and multiple MLNs, etc., also contributed to the poor survival.

\section{Conclusion}

Mesorectum MLNs from cervical cancer are rare. And, the survival of patients with mesorectum MLNs was poor after definitive concurrent chemoradiotherapy, although the mesorectum MLNs were boosted to 59-61Gy.

\section{Ethics Statement}

The Institutional Review Board (IRB) of Peking Union Medical College Hospital reviewed and approved the protocol. The ethical committee approval number is S-K398. Written informed consent from the study subjects was not acquired because: the research involves no more than minimal risk to the subjects; the waiver or alteration will not adversely affect the rights and welfare of the subjects; this is a retrospective study, the research could not practicably be carried out without the requested waiver or alteration. We confirm that patient data confidentiality is protected strictly. There is no identifiable information in the paper.

\section{Data Sharing Statement}

The datasets used and analyzed during the current study are available from the corresponding author on reasonable request.

\section{Author Contributions}

All authors contributed to data analysis, drafting or revising the article, gave final approval of the version to be 
published, and agree to be accountable for all aspects of the work.

\section{Funding}

This study is supported by the Ministry of Science and Technology of the People's Republic of China (grant number 2016YFC0105207).

\section{Disclosure}

The authors declare that they have no competing interests in this work.

\section{References}

1. Chen W, Zheng R, Baade PD, et al. Cancer statistics in China, 2015. CA Cancer J Clin. 2016;66:115-132. doi:10.3322/caac.21338

2. Liu Z, Hu K, Liu A, et al. Patterns of lymph node metastasis in locally advanced cervical cancer. Medicine (Baltimore). 2016;95:e4814. doi:10.1097/MD.0000000000004814

3. Li X, Yin Y, Sheng X, et al. Distribution pattern of lymph node metastases and its implication in individualized radiotherapeutic clinical target volume delineation of regional lymph nodes in patients with stage IA to IIA cervical cancer. Radiat Oncol. 2015;10:40. doi:10.1186/s13014-015-0352-5

4. Huang $\mathrm{H}$, Liu J, Li Y, et al. Metastasis to deep obturator and para-aortic lymph nodes in 649 patients with cervical carcinoma. Eur J Surg Oncol. 2011;37:978-983. doi:10.1016/j.ejso.2011.08.128
5. Barrett OC, McDonald AM, Lee Burnett O 3rd, Kim RY. Mesorectal node metastasis from gynecological cancer in the era of 3D conformal pelvic radiation therapy and intensity modulated radiation therapy. Pract Radiat Oncol. 2016;6:402-404. doi:10.1016/j. prro.2016.03.008

6. Wang W, Meng Q, Hou X, et al. Efficacy and toxicity of image-guided intensity-modulated radiation therapy combined with dose-escalated brachytherapy for stage IIB cervical cancer. Oncotarget. 2017;8:102965-102973. doi:10.18632/oncotarget.22434

7. Wang W, Hou X, Yan J, et al. Outcome and toxicity of radical radiotherapy or concurrent chemoradiotherapy for elderly cervical cancer women. BMC Cancer. 2017;17:510. doi:10.1186/s12885017-3503-2

8. Pecorelli S. Revised FIGO staging for carcinoma of the vulva, cervix, and endometrium. Int $J$ Gynaecol Obstet. 2009;105:103-104. doi:10.1016/j.ijgo.2009.02.012

9. Kasuya G, Toita T, Furutani K, et al. Distribution patterns of metastatic pelvic lymph nodes assessed by CT/MRI in patients with uterine cervical cancer. Radiat Oncol. 2013;8:139. doi:10.1186/ 1748-717X-8-139

10. Liu B, Gao S, Li S. A comprehensive comparison of CT, MRI, positron emission tomography or positron emission tomography/CT, and diffusion weighted imaging-MRI for detecting the lymph nodes metastases in patients with cervical cancer: a meta-analysis based on 67 studies. Gynecol Obstet Invest. 2017;82:209-222. doi:10.1159/ 000456006

11. Choi HJ, Ju W, Myung SK, Kim Y. Diagnostic performance of computer tomography, magnetic resonance imaging, and positron emission tomography or positron emission tomography/computer tomography for detection of metastatic lymph nodes in patients with cervical cancer: meta-analysis. Cancer Sci. 2010;101: 1471-1479. doi:10.1111/j.1349-7006.2010.01532.x

\section{Publish your work in this journal}

Cancer Management and Research is an international, peer-reviewed open access journal focusing on cancer research and the optimal use of preventative and integrated treatment interventions to achieve improved outcomes, enhanced survival and quality of life for the cancer patient.
The manuscript management system is completely online and includes a very quick and fair peer-review system, which is all easy to use. Visit http://www.dovepress.com/testimonials.php to read real quotes from published authors. 